\title{
A Spatial Smoothing Formulation for Location Systems
}

\author{
JOSÉ M. F. MOURA, MEMBER, IEEE
}

(Invited Paper)

\begin{abstract}
Two extensions of the classic passive location problem are considered. The first examines the ranging ability of passive receivers. The second presents alternative ways of describing the geometric content in positioning problems. The implications of the different approaches on the structure and performance of the location receiver are discussed.

Because range determination by passive means is missing, the classical formulation of passive location may be viewed as a local geometry demodulation problem. With the explicit consideration of range, passive location becomes a global problem. At stake is what may be gained by processing the small but valuable amount of information carried by the wavefront curvature of the signals. Relevant questions relate to the design of passive receivers that aptly demodulate the range and the remaining quantities defining the geometry.
\end{abstract}

There are passive applications where models exhibiting a high degree of (geometric) regularity are viable from a practical point of view. These occur, for example, when one can assume that the array sensors are collinear and that the moving target follows a deterministic linear path. In these models, the geometry is completely determined by a finite set of (unknown) parameters (e.g., range, bearing, speed, etc.). Accordingly, it is said that the regular models use an integral or ensemble approach for the description of the geometry. In many other problems, the geometry is more adequately deseribed by statistical processes. Examples arise when the source follows a disturbed path, or when, due to towing, the array shape deforms, acquiring a not-completely-known shape. The paper models these constraints: via a set of stochastic differential equations. The resulting representation is termed a differential description. It is emphasized that the differential approach is not only applied to the time content (relative dynamics), but also to the spatial dimension (array shape). The technique dualizes the space and time aspects of the problem. It provides a more flexible framework than the previous one. More gèneral motions and array shapes than the traditional collinear ones can be considered by the analysis, e.g., irregular line arrays or arrays where the sensors are located at positions with a certain degree of randomness.

Each approach fits a different design framework. The ensemble description is associated with the maximum likelihood technique. The differential representation uses recursive estimation methods (as provided by the Kalman-Bucy filtering theory). The paper discusses the main aspects of the structure of the resulting receivers and the associated measures of error performance. A second advantage of the differential model is immediately apparent. The recursiveness of the differential receiver reduces its computational load. The speed-up obtained is fully appreciated in tracking applications, where the observations are sequentially updated. Finally, it is interesting to note that the time/space duality provided by the differential approach exhibits a remarkable distinction: the location recursive receiver behaves in time as a filter, while it behaves in space as a smoother.

Manuscript received December 23, 1982 ; revised May 25, 1983. This work was partially supported by the JNICT under Research Contract $118 / 79-82$ and by INIC.

The author is with CAPS Complexa I, Instituto Superior Técnico, P-1096 Lisboa Codex, Portugal.

\section{INTRODUCTION}

IN ARRAY PROCESSING, the classical problem consists in the presence of a target being detected at a certain bearing. If the receiver is passive, this is accomplished by processing the received signature of the target. The approach constrains the geometry of the problem, reducing significantly the number of unknowns specifying it. Traditional contexts are as follows:

i) The bearings-only problem. The point source is not moving. and the receiver's line array is geometrically linear. The geometry is fixed by the unknown bearing $\theta$ of the source with respect to the receiver baseline. Other parameters defining the overall geometry, like the source/receiver separation, are ignored in this step of the processing.

ii) The bearing and Doppler problem. The point source moves with a constant speed $v$ along a deterministic linear track which is radial with respect to the geometric center of the receiver's linear array. The determination of the geometry requires the estimation of $\theta$ and $v$.

In both problems, the so-called far-field assumption is made, i.e., the wavefront of the received signal at the receiver site is taken to be planar. The location system has no passive ranging ability. Range is determined by alternative means (e.g., with an active sonar). The two quantities $\theta$ and $v$ are assumed time invariant and deterministic (albeit unknown). The location receiver essentially correlates replicas of the received signal. These are obtained at sensors with different spatial locations (spatial diversity), or at different time instants (temporal diversity). In other words, the correlation methods explore the signal's structure to find out the relative delays. From these, the estimates of the unknown parameters are generated by a geometry conversion. At each time instant, the relative propagation delay between two different sensors $s_{1}$ and $s_{2}$ is

$$
\tau_{s}=(\cos \theta) d / c
$$

where

$$
d=\left\|s_{1}-s_{2}\right\|
$$

is the baseline defined by $s_{1}$ and $s_{2}$, and $c$ is the medium propagating speed. At the reference sensor, the relative delay induced by the source motion between two different instants $t_{1}$ and $t_{2}$ is

$$
\tau_{t}=v\left(t_{1}-t_{2}\right) / \mathrm{c}
$$

The receiver accomplishes the estimation of $\theta$ and $v$ from 
the estimates of $\tau_{s}$ and $\tau_{t}$. As seen, the bearing $\theta$ is specified by processing the spatial structure of the received signals (1.1), while the speed $v$ is determined by processing the time structure as enriched by the Doppler shifts (1.3). In this setup, the location problem becomes conceptually simple. It is emphasized, that this simplicity is a result of the geometry constraints assumed; namely, the planar wavefront (far field), the linearity of the source track, and the collinearity of the receiver baseline array.

The present paper considers generalizations of the above problems along two different directions. The first relates to the study of the ranging ability of the passive receiver. The second considers more realistic positioning problems. These will include the situation where the source motions are not deterministic (e.g., as when random accelerations disturb the target dynamics), and the instance where the shape of the receiver line array is not completely known a priori (e.g., as when the locations of the sensors have some uncontrolled degree of uncertainty).

The first generalization lifts the far-field assumption. The wavefront curvature provides a small but valuable amount of information about the range separating the source and the receiver. As a direct consequence of the higher number of unknowns to be resolved, the location receiver becomes more complex. Also, contrasting with the neat decoupling of (1.1) and (1.3), the wavefront curvature couples the signal space and time structures. The receiver is studied in Section III. The approach to be taken generalizes the beam form concept. The receiver searches the space of the unknown parameters (two bearing angles, speed, range) in an optimal way, being designed by the application of the maximum-likelihood (ML) technique. The ML method extends to this multiparameter passive problem the ambiguity function approach of the Doppler radar context. The study can be carried out quite far [1]-[3], providing insight on the structure of the ML receiver and leading to analytical formulas that quantify its mean-square-error performance.

Underlying the ML approach is the possibility of representing the geometry by a finite set of unknown parameters. From a practical point of view, this is adequate whenever array misalignments or motion perturbations are not significant. However, collinearity is hard to guarantee in passive ranging applications. To measure the curvature of the wavefront, one has to resort to very-large baseline arrays or to listen to the target over extended periods of time, during which the source certainly maneuvers and deviates from its linear track. Also, in towed array applications, the array shape deforms significantly, assuming an irregular pattern which may not be completely known to the receiver. The ML approach is quite sensitive to the perturbations not accounted for by the model. These limit the attainable quality of the range estimate which may be below the desired level of performance.

The paper proposes a second generalization, which departs radically from the classical approach. The motions are modeled as a statistical process. They are described by a set of stochastic ordinary differential equations (SODE). The derivatives are with respect to the time parameter. These equations result by direct application of Newton's law of motion. They describe the dynamics of the point target relative to the array. The same methodology is applied to describe the shape of the line array by a set of SODE's. The derivatives are taken with respect to the spatial parameter (arc length). The equations are obtained by application of elementary methods drawn from differential geometry. This completely dualizes the time and space aspects of the problem. The receivers are designed via the theory of stochastic recursive filtering e.g., the KalmanBucy optimal linear filter. In general, the problem is nonlinear, the design requiring complex techniques. The SODE approach is presented in Sections IV and V. Section IV explores the stochastic nature of the time aspects of the problem. Section $\mathrm{V}$ concentrates on the novel issues arising when describing the array geometry via a set of SODE's. The SODE approach is named the differential description, while the classical one is called the integral or ensemble approach.

Setting the problem within the structure of recursive filtering, the differential description provides for:

i) A more robust framework, since the motions and/or the array shape become statistical processes. This loosens the tight geometry constraints that have to be assumed by the integral approach. The location receiver becomes less sensitive to path instabilities and to irregularities of the array shape.

ii) More general geometries. For example, the array elements do not have to be collinear. They may form an irregular pattern. The problem lies in finding the corresponding equations describing it.

iii) A reduction of the computational effort associated with the location receiver. This is a consequence of the recursive nature of the Kalman-Bucy type receiver. This point becomes of importance in tracking environments. Because the ML receiver is not recursive, the computations have to be completely redone whenever a new data point is available. With the Kalman-Bucy filter, the estimates of the source location are simply updated through the processing of the new data without unnecessary reprocessing of the previously obtained data points.

Besides the differences in the algorithm design techniques, it is worthwhile to note that the performance studies also employ different procedures. A usual measure of quality for the ML estimator is based upon the Cramér-Rao inequality. With the differential approach, the covariance of the meansquare error requires integration of a nonlinear differential equation of the Riccati type.

A final important remark is in order. When one goes from the integral to the differential description, the correct conceptual formulation along the time domain is that of a filtering problem (construction of the estimate at the present epoch, using all past measurements). Along the space domain, it is that of a smoothing processor. Smoothing means estimation with respect to an intermediate point of the data span. In fact, it usually makes more sense to construct the estimate of the source position with respect to an interior point of the array (e.g., its geometric center) than with respect to one of its extremities (e.g., its right end). This point is related to the classical question of which array sensor should be taken as reference when localizing noisy sources. 


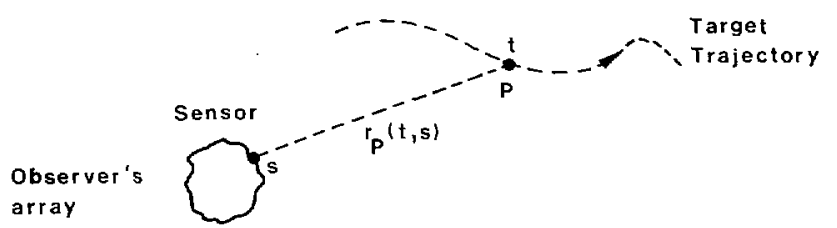

Fig. 1. General geometry for the positioning problem: A line array of irregular shape tracking a moving point source $P$.

\section{THE POSITIONING PROBLEM}

Fig. 1 shows a general planar geometry, where the source is a point. The positioning vector $r_{P}(t, s)$ is parameterized by the time instant $T$ and the sensor's location $s$. Although only the continuous array problem will be treated in the paper, point $s$ of the array will be indiscriminately referred to as the array element $s$ or the location $s$ of the array. In three-dimensional space, $s$ is a vector of three coordinates, which may be themselves time dependent.

Due to a variety of physical phenomena, e.g., fading, multipath, scattering, the transmission channel (such as for example, the underwater acoustics channel), is a complex system. Here, a simplifying point of view is taken, the action of the channel being reduced to a propagation delay and to the addition of a corrupting noise. The wavefield corresponds to a propagating signal $y(t)$. At time $t$ and sensor $s$, the received waveform $z(t, s)$ is

$$
z(t, s)=y(t-\tau(t, s))+w(t, s), \quad t \in T, s \in L .
$$

The delay $\tau(t, s)$

$$
\tau(t, s)=\left\|r_{P}(t-\tau(t, s))\right\| / c, \quad t \in T, s \in L .
$$

which is approximated by

$$
\tau(t, s) \cong\left\|r_{P}(t, s)\right\| / c, \quad t \in T, s \in L .
$$

Equation (2.3) is a reasonable approximation if $\left\|d r_{P} / d t\right\| / c \ll$ 1 , i.e., the target speed is significantly smaller than the wave propagation speed $c$. The observation interval is $T$, and the array definition domain is $L$. The positioning vector $r_{P}$ locates the source with respect to the sensor $s$.

It is seen from (2.1) and (2.2), that the geometry $r_{P}(t, s)$ modulates the received signal through the delay $\tau$. The task of the positioning processor is to reconstruct the geometry, i.e., to determine the vector $r_{P}$ for every $t \in T$ and $s \in L$, from the wavefield window (2.1). Although more complex signal and noise structures can be considered, the paper assumes that the signal is narrow-band-a single tone-and that the additive noise is wide-band, being uncorrelated from sensor to sensor. The focus of the work is on the geometry aspects. Geometry means here the description of $r_{P}(t, s)$ and the definitions of $T$ and $L$. For point sources, these descriptions depend on:

i) the shape and dynamics of the observer's array,

ii) the motions of the target.

In what follows, the processor is viewed as accomplishing two tasks: a differential delay or Doppler demodulation, and

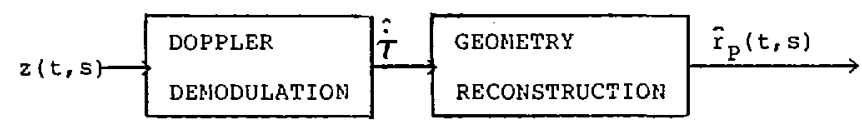

Fig. 2. Conceptual decomposition of the location receiver: Doppler demodulation followed by a geometry conversion.

a geometry reconstruction; see Fig. 2. In general, this block decomposition is solely conceptual, i.e., the optimal receiver does not decouple as suggested by Fig. 2. However, there are problems of practical significance for which the performance of the optimal receiver is indeed a factor. Examples of these are the narrow-band signal case [1]-[3], and the large timebandwidth product application [4]. The decomposition of Fig. 2 is also useful because it provides guidelines concerning the basic issues underlying the study of the positioning receiver. As a rule, the nature of the signal and noise processes influences directly the differential delay or Doppler estimation, while the array shape and relative motions strongly affect the geometry conversion block.

The purpose of the paper is to consider different ways of describing the geometry constraints and to analyze the impact of these different methods on the structure of the receivers and on their performance. The following sections will develop the integral and differential approaches. The analysis is pursued for three different contexts:

i) The first uses an integral description for the time (dynamics) and the space (array shape) aspects of the problem. This class is a generalization of the classic problem of array processing (bearings only) to simultaneous range, bearing, and speed determination. The integral formulation reduces the location problem to the estimation of a finite number of unknown quantities. The ML receiver generalizes the classical beam formers, providing an asymptotically efficient estimator. It measures the spherical curvature of the received wavefields, being capable of globally demodulating the geometry. Its nonrecursiveness penalizes, however, its applicability to tracking situations.

ii) The second utilizes an integral description for the space constraints and a differential representation for the motions. The resulting receiver exhibits a hybrid strategy. ML techniques explore the spatial contents of the signal to yield the estimation of part of the geometry. Recursive filtering tracks the time variations of the geometry.

iii) The third describes, in differential form, both the space and time structure of the geometry. The receiver is recursive in both dimensions. As will be noted, it behaves as a filter in the time domain and as a smoother in the spatial dimension.

\section{INTEGRAL SPACE/INTEGRAL TIME DESCRIPTION: MAXIMUM-LIKELIHOOD APPROACH}

Fig. 3 shows a general positioning geometry. The source moves with constant speed along a linear track. The array is geometrically linear. As formulated in the figure, the positioning system locates the source relative to the receiver. Hence, the array motions are immaterial to the relative geometry demodulation problem [3]. The constraints of a linear track and of a linear array shape lead to the following analytically closed 


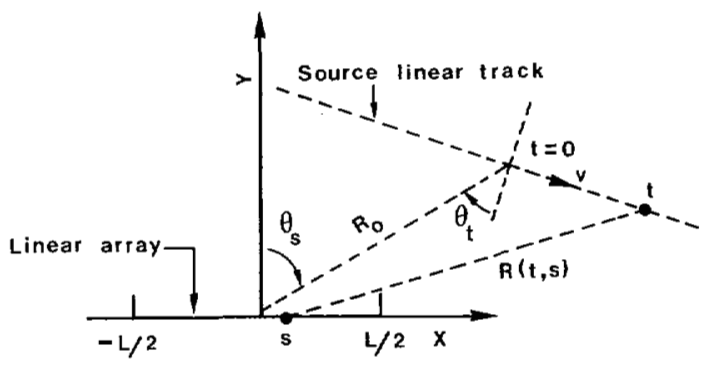

Fig. 3. Space/time integral geometry: Linear array tracking a constant speed undisturbed source. $R_{0}=$ reference range, $\theta_{s}$ and $\theta_{t}=$ space and time bearings, $v=$ speed.

form for the source-receiver separation

$$
\begin{aligned}
R(t, s)= & \left\{\left[R_{0}-s \sin \theta_{s}-v t \sin \theta_{t}\right]^{2}\right. \\
& \left.+\left[s \cos \theta_{s}+v t \cos \theta_{t}\right]^{2}\right\}^{1 / 2} .
\end{aligned}
$$

The observation interval $T=[-T / 2, T / 2]$, and the array domain $L=[-L / 2, L / 2]$ are intervals of the real line. The geometry is described by four unknown quantities, which are collected in

$$
A=\left[R_{0}|v| \sin \theta_{t} \mid \sin \theta_{s}\right]^{T}
$$

This generalizes the bearings-only problem, for which the unique quantity that is estimated directly from the passive measurements is the spatial bearing $\sin \theta_{s}$. It also includes two other important applications: the nonmoving source configuration [1] $(v=0)$, and the moving source/omnidirectional observer problem [2]. When the source does not move, the description of the relative geometry requires solely two quantities

$$
A_{s}=\left[R_{0} \mid \sin \theta_{s}\right]^{T}
$$

i.e., the range $R_{0}$ and the spatial bearing $\sin \theta_{s}$. For this problem, the source-receiver separation $R(s)$ is only a function of the domain $s$ (spatial diversity), being homogeneous in $t$. When the source moves, but the observer is omnidirectional, as is the case when the array is reduced to its reference sensor, there are three unknown quantities

$$
A_{t}=\left[R_{0}|v| \sin \theta_{t}\right]^{T} .
$$

The separation $R(t)$ is now independent of $s$ (time diversity). In (3.3) and (3.4), the subscripts $s$ and $t$ emphasize the nature (space and time) of the available baselines. The original problem described by (3.1) and (3.2) considers, as already mentioned, that the source moves and that the observer has a directional linear array. It is a coupled problem. The separation $R(t, s)$ exhibits a spatial diversity (linear array) and a time baseline. This temporal diversity is synthetically generated by the source dynamics.

For problems of the above type, the positioning receiver generalizes the beam-former processor of the classical bearingsonly context. It is designed by ML techniques. The ML processor is a correlator device in the space $\Omega$ of the parameter vector $A$, followed by a square-law device (fading channel). The estimate of $A$ corresponds to the maximum in $\Omega$ of the log- likelihood function. This function is designated the generalized ambiguity function (GAF) [1] - [3]. It presents a peaked structure. For sufficiently large $\Omega$, it exhibits secondary peaks of strength up to 10 to 20 percent of the main peak in $\Omega$. In practical terms, the maximization in the space $\Omega$ is accomplished in steps. The space $\Omega$ is divided into discrete segments by a succession of lattices of diminishing meshes, each one narrowing the resulting expected error. Limiting factors of the receiver behavior are evaluated from:

i) the strength of the main lobe relative to the secondary maxima of the ambiguity function;

ii) the quadratic structure of the main lobe, as approximately described by its second-order type expansion in $\Omega$, about the true actual value of the vector $A$.

In [1]-[3], the structure and performance of the receiver have been extensively studied for the case of a fading channel. Using the closed-form formulation of (3.1), the analysis therein remains valid for arbitrary values (large and small) of the time and space baselines, i.e., of the geometric ratios

$$
X_{t}=v T /\left(2 R_{0}\right)
$$

and

$$
X_{s}=L /\left(2 R_{0}\right) .
$$

The ML theory provides insight into the expected behavior of the receiver, suggesting the compromises between the error performance, the geometry (baseline $L$ or $v T$ versus $R_{0}$ ), and the noise environment (signal-to-noise ratio (SNR)). For arbitrary conditions, the quantitative performance results are best displayed by graphics. For different geometric conditions and statistical noise environments, several curves are available in [1]-[3]. To obtain analytical formulas that bound the mean-square error associated with each component of $A$, one has to resort to limiting arguments in $X_{t}$ and/or $X_{s}$. For small values of these parameters, one proceeds with a truncated Taylor series. For large values of $X_{t}$ and $X_{s}$, one pursues asymptotic expansions in these parameters. To illustrate the type of results that are obtained, the Cramér-Rao bound for the range $R_{0}$ is indicated for each one of the three problems mentioned above, when $X_{s}<1$ and $X_{t}<1$. The standard deviation is given by:

\section{nonmoving source}

$$
\sigma_{R}=G^{-1 / 2}(\lambda / 2 \pi)\left[3 \sqrt{5} /\left(\cos \theta_{s} X_{s}^{2}\right)\right] .
$$

moving source/omnidirectional observer

$$
\sigma_{R}=G^{-1 / 2}(\lambda / 2 \pi)\left[5 \sqrt{7} /\left(\sin \theta_{t} \cos \theta^{2}{ }_{t} X_{t}{ }^{3}\right)\right]
$$

moving source/linear array

$$
\sigma_{R}=G^{-1 / 2}(\lambda / 2 \pi)\left[3 \sqrt{5} /\left(\cos \theta_{s} X_{s}^{2}\right)\right] p(\gamma) .
$$

In (3.9), the rational function $p(\gamma)$ is

$$
p(\gamma)=\left(1+4 \gamma^{2} / 5\right) /\left[1+\left(4 \gamma^{2} / 5\right)+\gamma^{4}\right]
$$


where

$$
\gamma=X_{c t} / X_{c s}=v T \cos \theta_{t} /\left(L \cos \theta_{s}\right)
$$

In the preceding expressions, $G$ is a gain dependent on the ratio of the average signal energy $\bar{E}_{r}$ to the noise level $N_{0}$. For narrow-band signals, uncorrelated noise and fading channels

$$
G=2\left(\bar{E}_{r} / N_{0}\right) \bar{E}_{r} /\left(N_{0}+\bar{E}_{r}\right)
$$

where $N_{0} / 2$ is the white noise statistical level. For the nonmoving source problem, (3.7) shows that the range estimation depends on second-order effects $\left(X^{2}\right)$ of the signal curvature, while for the moving source/omnidirectional observer problem it depends on third-order effects $\left(X^{3}\right)$. On the other hand, (3.9) shows how coupling affects the range estimation performance. For similar expressions concerning the standard deviation of the error of the other components of $A$, the reader is referred to [1]-[3].

The drawbacks of the ML formulation of the passive positioning problem are its nonrecursive nature, and the difficulties in applying it to more realistic models. Being nonrecursive, the ML processor may require a large computational effort. At each step of the search algorithm, this effort relates to the number $M$ of evaluations of the $\log$-likelihood function. The value of $M$ is given by

$$
M=V_{A} / V_{\text {cell }} .
$$

In (3.13), $V_{A}$ is the volume of the region of the space $\Omega$ of the parameter vector $A$ which has to be scanned in order to find the true location of the source. This is the region of $a$ priori uncertainty. $V_{\text {cell }}$ is the volume of each basic cell of the search lattice. For the first step of the search procedure, a good compromise is to divide into discrete segments the $a$ priori uncertainty region with a lattice of mesh at most as large as the dimensions of the main lobe of the ambiguity function. This assures that at least a vertex of the lattice lies on the main lobe. With high probability, for weak noise conditions, the first step will then choose the right cell. To obtain an estimate of $V_{\text {cell }}$, the main lobe is approximated by a quadratic expression in the space $\Omega$. If $m$ is the matrix with respect to the components of $A$, the volume $V_{\text {cell }}$ is inversely proportional to the square root of the determinant of $m$ [3]. One obtains

$$
M \sim k V_{A}(\operatorname{det} m)^{1 / 2} .
$$

Figs. 4 and 5 show the behavior of $\operatorname{det} m$ for the nonmoving source and the moving source/omnidirectional observer problems, respectively. They assume that $R_{0}=10^{4} \mathrm{ft}(\approx 3 \mathrm{~km})$. The figures also show formulas for the limiting behavior of det $m$ (i.e., for small and large values of the geometric ratio $X$ ). For small $X_{s}$ and $X_{t}$ (left-end asymptotes), after rearrangement, one can arrive at the following expressions for the number $M$ of required evaluations of the log-likelihood function:

\section{nonmoving source}

$$
M_{s} \sim k\left[(2 \pi)^{2} /(3 \sqrt{15})\right]\left(R_{0} / \lambda\right)^{2} \cos ^{2} \theta_{s}\left(V_{A} / R_{0}\right) X_{s}^{3}
$$

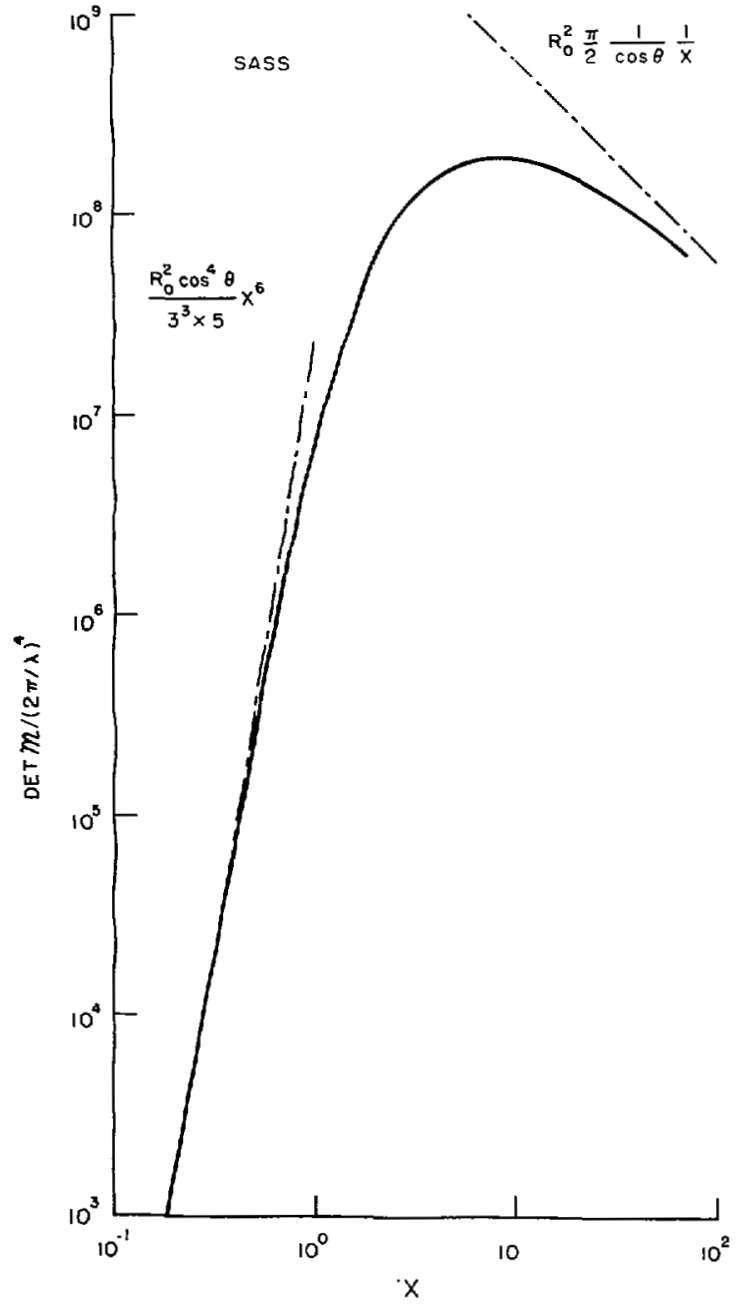

Fig. 4. Det $m$ as a function of the geometric ratio $X_{s}=L / R_{0}$, for the nonmoving source problem.

moving sourcelomnidirectional sensor

$$
\begin{aligned}
M_{t} \sim & k\left[2^{4} \pi^{3} /(15 \sqrt{105})\right]\left(R_{0} / \lambda\right)^{3} \sin \theta_{t} \\
\cdot & \cos ^{2} \theta_{t}\left[V_{A} /\left(v R_{0}\right)\right] X_{t}{ }^{6}
\end{aligned}
$$

moving source/linear array

$$
\begin{aligned}
M_{s t} \sim & (2 \pi / 3)^{4}(3 / \sqrt{5})\left(\cos \theta_{s} \cos \theta_{t}\right) n(\gamma)\left(R_{0} / \lambda\right)^{4} \\
& \cdot\left[V_{A} /\left(\nu R_{0}\right)\right] X_{s}{ }^{2} X_{t}^{4}
\end{aligned}
$$

where

$$
n(\gamma)=1+(4 / 5) \gamma^{2}+\gamma^{4}
$$

and $\gamma$ has been defined in (3.11). The constant $k$ depends on the specific form of the basic lattice cell (e.g., ellipsoidal, rectangular, etc.). Table I evaluates the number $M$ for different configurations. In the table, the triple sets represent the order of magnitude of the normalized values of $\left(M_{s}, M_{t}, M_{s t}\right) / k$. These values have been evaluated for $X_{s}=X_{t}=0.1, \theta_{s}=\theta_{t}=$ $45^{\circ}$. The values in the table show that the computational effort may be extremely large, exceeding the available resources. Of course, more sophisticated algorithms than the grid-search- 


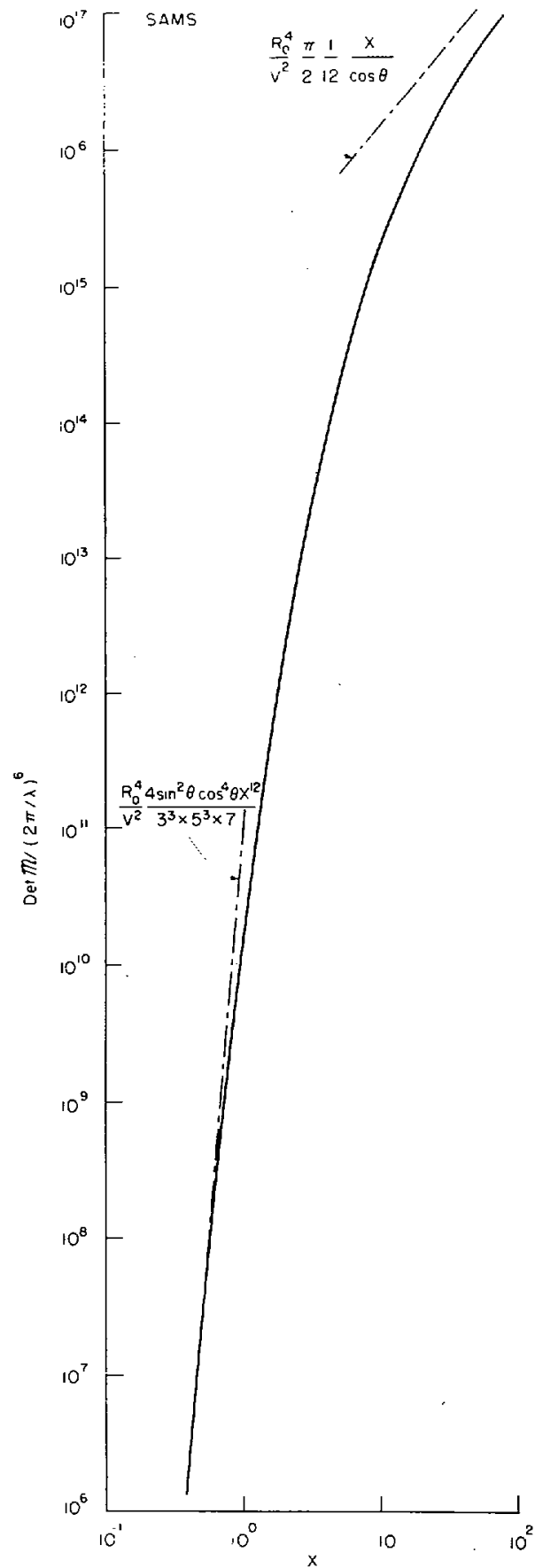

Fig. 5. Det $m$ as a function of the geometric ratio $X_{t}=v T /\left(2 R_{0}\right)$ for the moving source/omnidirectional sensor problem.

TABLE I

THE COMPUTATIONAL COMPLEXITY OF THE ML RECEIVER AS MEASURED BY THE NUMBER OF LOG-LIKELIHOOD FUNCTION EVALUATIONS $\left(M_{s}, M_{t}, M_{s t}\right)$ $\left(R_{0} / \lambda=\right.$ ratio of the range over the wavelength, $V_{A} /\left(u R_{0}\right)=$ volume of $a$ priori uncertainty normalized by the source parameters)

\begin{tabular}{|l|c|c|}
\hline$V_{A} / V_{0}$ & 1 & 10 \\
\hline 200 & $\left(40,10,3 \times 10^{4}\right)$ & $\left(4 \times 10^{2}, 10^{2}, 3 \times 10^{5}\right)$ \\
\hline 2000 & $\left(4 \times 10^{3}, 10^{4}, 3 \times 10^{8}\right)$ & $\left(4 \times 10^{4}, 10^{5}, 3 \times 10^{9}\right)$ \\
\hline
\end{tabular}

ing procedure, discussed earlier, may reduce the computational load. The numbers of the table, exhibiting the rapid growth of $M$ with $\left(R_{0} / \lambda\right)$ for the different problems, give a clear indication of how the computational effort increases with the complexity of the problem.

The nonrecursiveness of the algorithm penalizes particularly applications where the observations correspond to a sequence of data, which increases every sample interval. Since the ML receiver makes no direct use of the previous estimate [except for a reduction of the a priori uncertainty region; namely, of $\left.V_{A} /\left(v R_{0}\right)\right]$, the computations have to be completely redone whenever a new data point is available. In particular, in tracking situations, it is of interest to be able to update the estimates using a minimum of computational power. The next section presents a differential formulation for the time aspects of the problem. This is a more general model, in the sense that the target motions are now stochastic. Also, it turns out that this accomplishes the two objectives: the recursiveness of the processor, and the robustness of the model.

\section{INTEGRAL SPACE/DIFFERENTIAL TIME DESCRIPTION: HYBRID APPROACH}

The preceding section has shown how an integral description in both the time and space dimensions leads to a geometry which is completely described by a finite number of unknown quantities. This simplicity hides two difficulties of different type:

i) an excessive computational load,

ii) a model which is sensitive to the instabilities that possibly appear in the real world.

To overcome these drawbacks, a differential description is proposed for the time aspects of the problem. With respect to the parameterization of Fig. 6 , the source-receiver separation $R(t, s)$ is written as

$R(t, s)=\left\{R^{2}(t)+s^{2}-2 s R(t) \sin \theta(t)\right\}^{1 / 2}, \quad t \in T, s \in L$.

Besides the linearity on the observer's array shape, (4.1) assumes no other specific constraints on the motions of the point source. The source-disturbed path is described by the sourceinstantaneous polar coordinates $R(t)$ and $\theta(t)$. Other coordinates may be employed, e.g., the rectangular frame $(X(t)$, $Y(t)$ ). To describe the dynamics of the source, use is made of Newton's second law of motion. A system of differential equations results. For a constant nominal linear path disturbed by random accelerations, the source dynamics in rectangular coordinates is described by

$$
\begin{gathered}
\ddot{X}(t)=u_{X}(t) \\
\ddot{Y}(t)=u_{Y}(t)
\end{gathered}
$$

where ( $\left.{ }^{*}\right)$ stands for time derivative, $\left[u_{X}(t) \mid u_{Y}(t)\right]^{T}$ is the vector of random accelerations. In polar coordinates, an equivalent description is

$$
\ddot{R}(t)=\dot{\theta}^{2}(t) R(t)+u_{R}(t)
$$




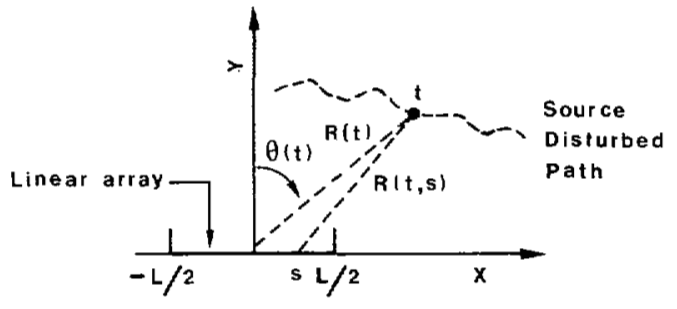

Fig. 6. Geometry for the positioning problem where a linear array tracks a source moving along a distributed path geometry.

$$
\ddot{\theta}(t)=-2 \dot{\theta}(t) \dot{R}(t) / R(t)+u_{\theta}(t) .
$$

Defining the state vector

$$
x(t)=[R(t)|\dot{R}(t)| \theta(t) \mid \dot{\theta}(t)]^{T}
$$

and the random vector

$$
u(t)=\left[u_{R}(t) \mid u_{\theta}(t)\right]^{T}
$$

the dynamics can be written as

$$
\dot{x}(t)=f[x(t), t]+u(t)
$$

with some unknown initial conditions. By making appropriate assumptions on the input noise, a meaningful statistical model results. For the same physical dynamics, (4.2) and (4.3) and (4.4) and (4.5), provide different modeling classes. The first set corresponds to a linear state-variable system, while the second set to a nonlinear one. In the sequel, the discussion uses the polar system.

For narrow-band and emitted signals, the received waveform is, in exponential notation,

$$
z(t, s)=\tilde{b} \exp j \phi(t, s)+w(t, s)
$$

where $b$ represents a fading gain. It is an appropriate model for slowly fading channels. According to Section II, the measurement noise $w(t, s)$ has a flat spectral density in the frequency band of interest, being uncorrelated from sensor to sensor. The phase is

$$
\phi(t, s)=W_{0} t-2 \pi R(t, s) / \lambda .
$$

Assuming that the array length $L$ is much smaller than the source-receiver separation, the function $R(t, s)$ is approximated by its first-order truncated Taylor series expansion

$$
R(t, s) \cong R(t)+\sin \theta(t) s, \quad s \in L, t \in T .
$$

Equation (4.11) represents a linearization in the $s$ domain of the wavefront end, leading to an instantaneous planar approximation of the wavefront curvature across the array. It is important to remark that (4.11) practically decouples the bearing estimation from the range determination. The angle $\theta$ is instantaneously estimated from the spatial diversity, while the polar distance is measured by processing the time sequential measurements; see Fig. 7.

The decoupling is suboptimal. It steers the array by se-

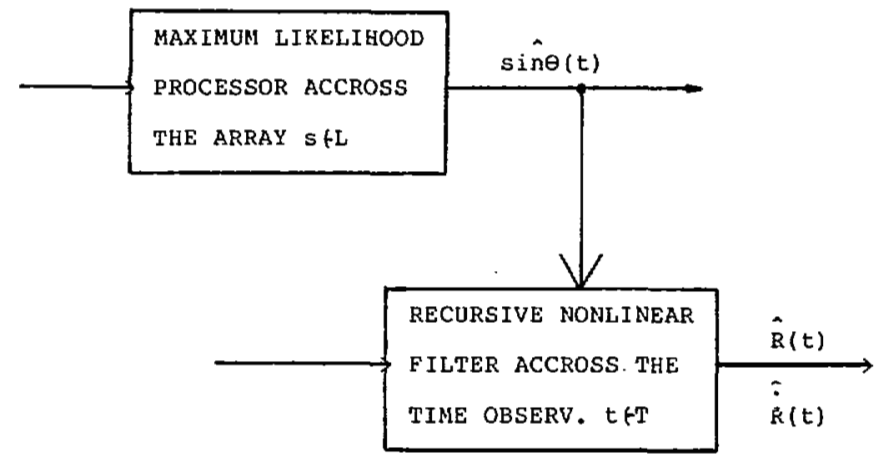

Fig. 7. Suboptimal decoupling of the location receiver: Angle and range estimation.

quentially updating the bearing through the ML processor in the $s$ dimension. This sequential updating is fed into the structure of the range filter. The latter is a nonlinear recursive filter. Recursive waveform filters can be designed by application of the Kalman-Bucy theory. This theory provides optimal solutions when the underlying models are linear. When nonlinearities are present, as in (4.4) and (4.9), the application of the theory requires linearization of the model nonlinearities. The resulting filter is the extended Kalman-Bucy filter (EKB). The EKB is well suited for tracking. For certain geometries and signal-to-noise ratios, it may exhibit initialization problems. This is reflected by the persisting bias on the range estimate. These initialization problems may be overcome by designing the filter via nonlinear filtering techniques; see [5] and references therein. The optimal filter has remarkable acquisition properties [6]. The difficulty lies in the computational complexity associated with the optimal nonlinear filter that makes it practical only for low-dimensional problems.

An alternative method has been presented in [7]. This approach uses a hybrid strategy. The hybrid algorithm switches between two modes of operation: an acquisition mode and a tracking mode. The first mode uses an ML processor designed via the techniques of the previous section. The ML filter acquires the source. Acquisition is accomplished when the initial global uncertainty in the source location and dynamics is resolved to within a prescribed value. This threshold is determined by the initialization requirements of the second mode. The length of the time interval needed for acquisition is referred to as the acquisition time $T_{\text {acq }}$. The second mode tracks the source dynamics. The tracking filter is recursive, corresponding to an EKB filter. It is initialized in agreement with the acquisition step. Relevant issues concerning the hybrid strategy relate to:

i) the sensitivity of the ML processor to the path perturbations;

ii) the nonlinear behavior of the linearized tracker.

The path perturbations, not being accounted for by the ML analysis, contribute to the mean-square error at the output of the receiver with an extra component $\Sigma_{R}^{2}$. For simplification, these two error components are assumed to be independent. The total mean-square error $\left(E_{R} M \mathrm{~L}\right)^{2}$ at the output of the $\mathrm{ML}$ receiver is then

$$
\left(E_{R}^{\mathrm{ML}}\right)^{2}=\sigma_{R}^{2}+\Sigma_{R}^{2} .
$$




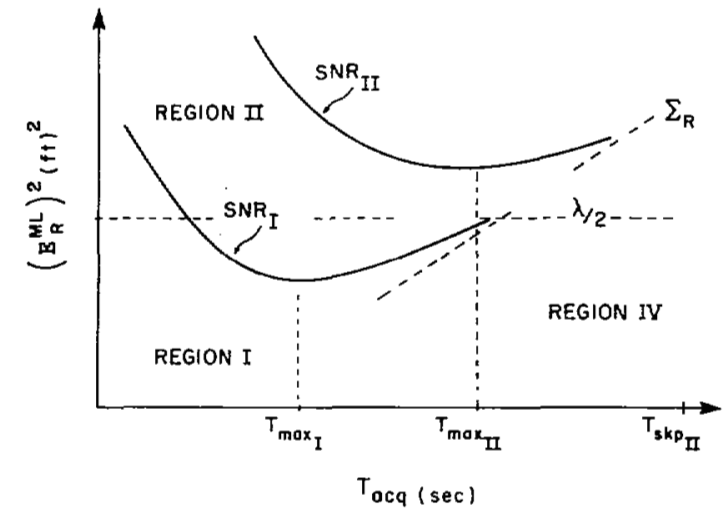

(a)

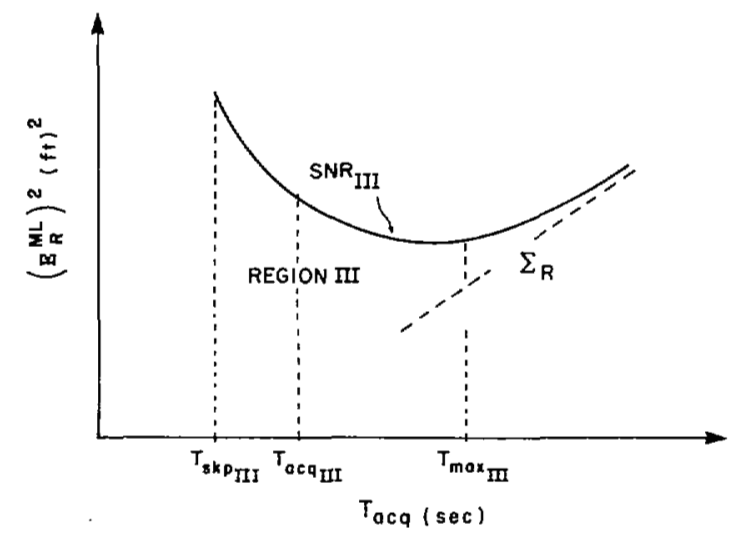

(b)

Fig. 8. Typical regions of behavior for the hybrid algorithm in positioning systems. $\left(E_{R} \mathrm{ML}\right)^{2}=$ total mean-square error at $\mathrm{ML}$ output, $\Sigma_{R}=$ component of mean-square error due to path perturbations, SNR $=$ signal-to-noise ratio, $\lambda=$ wavelength

The first component decreases monotonically with time (Cramér-Rao), while the second increases (typical behavior of diffusion processes). Their sum has the convex cup behavior depicted in Fig. 8 . The curves have a minimum which corresponds to the maximum time $T_{\max }$ that can be used during the acquisition step; i.e., one should take $T_{\text {acq }}$ such that

$$
T_{\text {acq }}<T_{\max } .
$$

In other words, $T_{\max }$ is an upper bound to the time during which the ML receiver can be used in a meaningful way.

Besides $T_{\text {acq }}$ and $T_{\text {max }}$, there is a third time limit $T_{\text {slp }}$ to be considered. This limitation is imposed on the hybrid strategy by the nonlinear behavior of the EKB filter. Phase trackers like the EKB and the phase-locked loop (PLL) are common circuitry in radio communication PM (phase-modulated) and FM (frequency-modulated) systems. Even for high SNR's, these systems exhibit from time to time a loss of lock, known as phase or cycle slips. These slips, usually identified as clicks, represent bursts of sudden jumps of the phase error process. These jumps correspond to an indeterminacy of several multiples of the wavelength on the range $R(t)$ (see (4.10) and (4.11)). In tracking problems, where distance is being measured by (a sort of) integration of the Doppler, these slips penalize permanently the error associated with the range estimate at the output of the EKB tracker. The error variance associated with the slip process increases with time at a certain diffusion rate. The parameter that characterizes the jump behavior is the average time to slip a cycle or the frequency of cycle slips. After the EKB filter has been initialized, the filter slips cycles at random from time to time. Its meansquare error degrades monotonically with time till it reaches an intolerable preset limit. The maximum elapsed time, being a random variable, has a mean value which is proportional to the mean time between jumps. Because the maximum time is related to the slip phenomenon, it is indicated here by $T_{\mathrm{s} 1 \mathrm{p}}$. After $T_{\text {slp }}$ seconds, the error at the tracker output exceeds the desired level of performance. The EKB has to be reinitialized by the ML processor. The three numbers $T_{\mathrm{acq}}, T_{\max }$, and $T_{\text {slp }}$ are nonlinearly dependent on the geometry and on the statistical environment, i.e., on the SNR. Contingent on the relation between these numbers, one can distinguish typical patterns of behavior for the hybrid strategy; see Fig. 8(a) and (b). These curves illustrate four typical regions. Regions I and II correspond to the situations where

$$
T_{\text {acq }}<T_{\max } \ll T_{\text {slp }} .
$$

This means that the ML step leads to the desired global accuracy, and that the EKB filter can be used for a useful interval of time, during which it filters and integrates the source dynamics. The two regions are distinguished by the value of the standard deviation $\sigma_{R}$ of the final error associated to the acquisition step.

In region $\mathrm{I}, \Sigma_{R}$ is smaller than half the wavelength $\lambda$, while in region II, it is greater. Region I is referred to as the tracking within the wavelength region, while region II corresponds to the tracking within the geometry region. In this latter case, at the output of the EKB filter, the range estimate is biased by a tolerable error. Region III is characterized by

$$
T_{\mathrm{slp}}<T_{\mathrm{acq}}<T_{\max }
$$

In this region, no use can be made of the EKB filter, since it loses lock in a time which is smaller than the acquisition time. Finally, region IV corresponds to

$$
T_{\max }<T_{\text {acq }} .
$$

In this case, the system has to be redesigned, either by using better sensors (improvement of their quality), or by inducing sensor motions, e.g., as with maneuvering of the obseryer's array, or by applying more sophisticated processors. This author [7] quantifies the qualitative discussion for several conditions of the underwater acoustics environment. For a relatively stable path, as when the power spectral level $q$ of the random accelerations (see (4.2) and (4.3)) is about $4 \times 10^{-3}$ $\mathrm{ft}^{2} / \mathrm{s}^{3}\left(\approx 0.37 \times 10^{-3} \mathrm{~m}^{2} / \mathrm{s}^{3}\right)$, cycle slipping is not a problem, even when the SNR is low ( $0 \mathrm{~dB}$ or under) (regions I and II). However, for larger random accelerations, as when the spectral level $q$ is $4 \mathrm{ft}^{2} / \mathrm{s}^{3}$, the working horizon $T_{\mathrm{skp}}$ of the EKB significantly determines the positioning processor performance. For this value of $q$ and when $\mathrm{SNR}=0 \mathrm{~dB}$, one can show that $T_{\max } \sim 3 \mathrm{~min}$ and $T_{\mathrm{sk}}$ is ten times as large. The tracker has to be reinitialized every half hour (region III). For a tenfold 
increase in the noise level (SNR $=-10 \mathrm{~dB}$ ), keeping the same random accelerations power $q, T_{\mathrm{max}}$ is slightly increased to $4 \mathrm{~min}$, but $T_{\mathrm{skp}}$ decreases significantly to $21 \mathrm{~s}$. The EKB is of no use, the algorithm reducing to its ML component (region III). This illustrates how, for the underwater acoustics environment, the geometric parameters and the SNR's are at critical levels. The designer may typically encounter underwater configurations for each of the above regions. See [7] for analytical and simulated studies.

\section{DIFFERENTIAL SPACE/DIFFERENTLAL TIME DESCRIPTION: RECURSIVE APPROACH}

In a natural way, Section IV has generalized the positioning framework to applications where more realistic dynamics can be considered. By a suitable reinterpretation of the array description, it is interesting to observe that the same techniques may be used in the spatial domain $s$. What results is a more general and robust class of location problems that includes arrays of irregular shape, as well as arrays where there is uncertainty about the exact location of the sensors. If the array is fixed, the impact of this problem of sensor location uncertainty may be reduced by determining the sensor's location with sources of known position. However, with towed arrays in the ocean, bending, stretching, and misalignment lead to complex shapes. Uncertainty is added. These effects are magnified by the disturbances that affect the motion of the towing ship. Also, with ocean bottom-moored sensors or with buoysuspended hydrophones, their precise location may be unknown.

The new framework has the added advantage that the receivers are of a recursive nature. It is emphasized that recursivity is now meant for both dimensions: time $t$ and space $s$ as well.

\section{Differential Description of the Line Array}

To obtain the differential description for the space aspects of the positioning problem, the array is, treated as a curve in space. To simplify the discussion, only the case of a line array lying on a plane will be studied. This plane is here called the reference plane. It contains the point source and its motions. As known from geometry, curves may be described by different parameterizations. In the usual language of the theory of curves, the word parameter is reserved to the independent variable used in the description of the curve, such as the time $t$ in trajectories of moving platforms. This contrasts with the meaning of unknown quantity given to the word parameter in Section III. In the following, its meaning will be transparent from the context.

To motivate the approach, let the line array be rigid with the shape shown in Fig. 9. It is an arc of a cardioid. The origin $O$ of the reference frame coincides with its cusp. The curve is represented by

$$
r=2 a(1-\cos \theta), \quad \theta \in U=[0, \pi] .
$$

Alternatively, differentiating this equation

$$
\begin{aligned}
& d r / d \theta=2 a \sin \theta, \quad \theta \in U[0, \pi] . \\
& r(0)=0,
\end{aligned}
$$

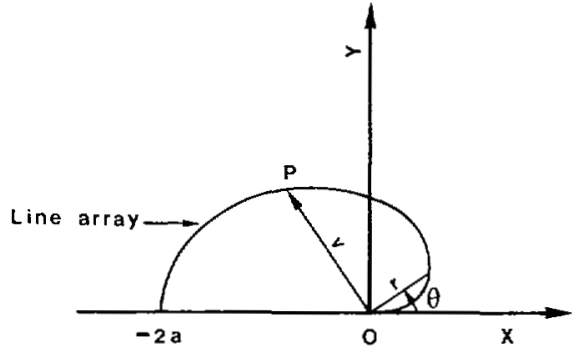

Fig. 9. Line array with the shape of a cardioid.

Equation (5.1) is what has been termed in Section III an integral or ensemble description for the line array. Equation (5.2) is a differential representation. The parameter used in both, i.e., the independent variable, is the polar angle $\theta$.

A trajectory of a line curve is the function $F(u)$, together with the interval $U$ on the real line $\operatorname{IR}^{1}$ where its defining parameter $u$ takes values. With respect to the above example, $F$ is

$$
\begin{aligned}
F: U=[0, \pi]\left(\mathbb{R}^{1} \rightarrow \mathbb{R}^{2}\right. \\
\theta \mid \rightarrow F(\theta)=(r(\theta), \theta) .
\end{aligned}
$$

In (5.3), $\mathbb{I}^{1}$ is the real line, and $\mathbb{I}^{2}$ is the plane. Equations (5.1) and (5.2) provide two different definitions for $F$. Both definitions use the parameter $\theta$ and the same interval $U$. In the differential description, the initial condition is part of the definition.

Let $v_{P}(u)$ represent the positioning vector of the running point $P$ of the trajectory

$$
v_{P}(u)=\left[\begin{array}{l}
F_{1}(u) \\
F_{2}(u)
\end{array}\right] .
$$

For the arc connecting the reference point $u_{0}$ to $u$, the arc length is

$$
s(u)=\int_{u_{0}}^{u}\|d v(u) / d u\| d u
$$

where $\|\cdot\|$ is the norm function. The arc length $s(u)$ is independent of the parameterization chosen. Under general conditions, a curve admits a representation in terms of its arc length $s$. From the practical side, this parameterization is the one that can be generalized in the simplest way. To see this, consider the line array represented in Fig. 10. From Fig. 10(a), a possible description is

$$
d Y / d X=0 \quad Y(0)=0, \quad X \in[-L / 2, L / 2] .
$$

A second possibility is

$$
\begin{aligned}
& d Y / d s=0 \\
& d X / d s=1
\end{aligned} \quad Y(0)=X(0)=0, \quad s \in L=[-L / 2, L / 2] .
$$




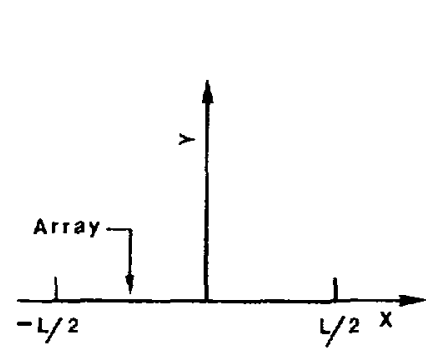

(a)

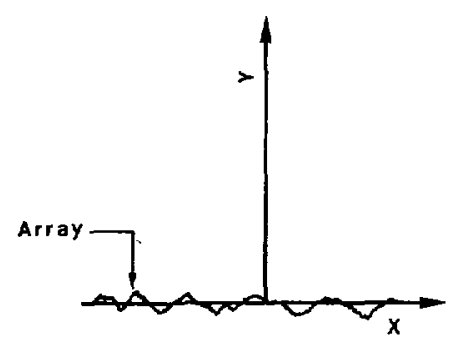

(b)
Fig. 10. Two examples of a linear (line) array. (a) Linear array. (b) Disturbed linear array.

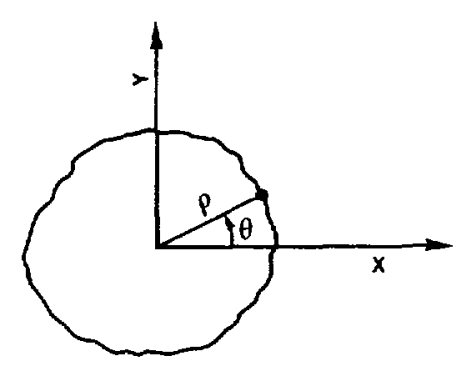

Fig. 11. Disturbed circle array.

To describe the disturbed linear array of Fig. 10(b), (5.7) is more naturally generalizable than (5.6). One obtains

$$
\begin{aligned}
& d^{2} Y / d s^{2}=u_{Y}(s) \\
& d^{2} X / d s^{2}=u_{X}(s), \quad s \in L=[-L / 2, L / 2] \\
& X(0)=0 \quad X^{\prime}(0)=0 \quad Y(0)=0 \quad Y^{\prime}(0)=0 .
\end{aligned}
$$

In (5.8), ( )' stands for the derivative with respect to the arc length parameter $s$. The random vector $u=\left[u_{X}, u_{Y}\right]^{T}$ models the uncertainty about the location of the array sensors. In the following, it will be assumed to be uncorrelated for different values of $s$ ("white noise"), i.e.,

$$
E u\left(s_{1}\right) \cdot u^{*}\left(s_{2}\right)=q I\left(s_{1}-s_{2}\right), \quad s_{1}, s_{2} \in L
$$

As done in Section IV, the position coordinates of the sensors and their derivatives up to a given order are collected in a state vector. For (5.8), a four-dimensional state vector is required

$$
x(s)=\left[X(s)\left|X^{\prime}(s)\right| Y(x) \mid Y^{\prime}(s)\right]^{T}
$$

The array shape is now determined by a state equation and an initial condition. The defining set $L$ of the array is simply an interval of the real line.

Equation (5.8) is an example of the linear class of state models

$$
\begin{aligned}
& d x(s) / d s=A(s) x(s)+B(s) u(s) \\
& x\left(s_{0}\right)=x_{0}, \quad s \in L .
\end{aligned}
$$

The array structure, i.e., the system matrices $A(s)$ and $B(s)$, may be known. If not, an identification procedure must be carried out, off-line or on-line. The elements of $A$ and $B$ can be $s$-dependent. The linear class (5.11) encompasses quite

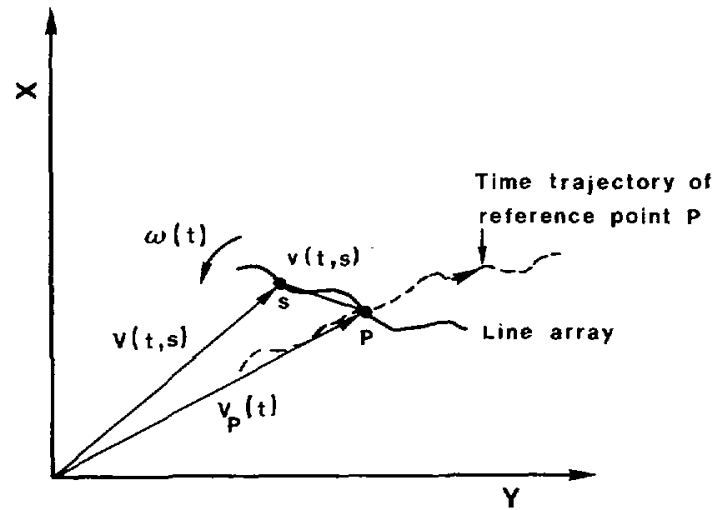

Fig. 12. Moving rigid array with respect to absolute frame.

general array shapes, not necessarily geometricaily linear. For example, using arguments similar to the ones above, the disturbed circle array of Fig. 11 is described by

$$
\begin{aligned}
& d^{2} \rho(s) / d s^{2}=u_{1}(s) \\
& d^{2} \theta(s) / d s^{2}=u_{2}(s), \quad s \in L \\
& \rho(0)=R \quad \rho^{\prime}(0)=0 \quad \theta(0)=0 \quad \theta^{\prime}(0)=1 / R .
\end{aligned}
$$

The more general state variable class is the nonlinear class

$$
\begin{aligned}
& d x(s) / d s=f[x(s), s]+g[x(s), s] u(s) \\
& x\left(s_{0}\right)=x_{0}, \quad s \in L
\end{aligned}
$$

where $f$ and $g$ are nonlinear vector functions satisfying appropriate conditions to ensure the existence and uniqueness of solutions to (5.14), i.e., that guarantee that (5.14) is a suitable mathematical model. To write $(5.14)$ properly involves the definition of stochastic integrals, and the use of the so-called Itô stochastic calculus. This is avoided here by interpreting (5.14) formally. Equation (5.14), as well as (5.12), describes the array shape. It also leads to the position coordinates of the sensors with respect to a frame coincident with the array itself. This will now be generalized to the case where the rigid array is moving.

\section{Rigid Array Motions}

To simplify the discussion, the motions are restricted to the reference plane. The dynamics of a rigid body can be decomposed in a translation and a rotation. Referring to Fig. 12, let $(X, Y)$ be an absolute frame $F, V_{P}(t)$ and $V(t, s)$ be the instantaneous position vectors at time $t$ with respect to the frame $F$ of the reference point $P$ of the line array and of the general sensor element $s$, and let $v(t, s)$ be the position vector of element $s$ with respect to $P$. From Fig. 12

$$
V(t, s)=V_{p}(t)+v(t, s), \quad t \in T, \quad s \in L .
$$

Time differentiation leads to

$$
d V / d t=d V_{P} / d t+d v / d t
$$


with

$$
d v / d t=W_{z} \wedge v
$$

where $W_{z}(t)=W(t) e_{z}$, and $W(t)$ is the angular rate of rotation with respect to $P$, and $\wedge$ stands for the external vector product. Equations (5.16) and (5.17) have used the rigid assumption on the line array. Higher order derivatives of (5.15) are constructed by observing that derivatives of rotating bodies with respect to fixed frames follow the rule

$$
\left.()_{F}(.)=\left\{()^{*}\right)+W_{z} \wedge\right\}(.) \text {. }
$$

For example, if the motion's description requires second-order time derivatives, application of (5.18) leads to

$$
d^{2} V / d t^{2}=d^{2} V_{P} / d t^{2}+W \wedge v+W \wedge(W \wedge v) .
$$

Equations (5.15) to (5.19) reflect the special structure assumed; namely, that of a rigid array. Because of this, time derivatives and spatial derivatives do not couple.

Using the techniques of Section IV, the disturbed motions of the array reference point $P$ may be modeled by a stochastic differential equation in $t$. Also, the instantaneous angular frequency may be described by a lumped state model. For a second-order process $W(t)$, a suitable model is

$$
\begin{aligned}
& \ddot{W}(t)+a W(t)=u_{W}(t) \\
& W\left(t_{0}\right)=W_{0} .
\end{aligned}
$$

In summary, the general problem of a line array of irregular and uncertain shape, moving through a disturbed path on a plane requires the following setup:

i) Array shape equations. For a rigid array, two differential equations are required, one in $s(5.14)$ and the other in $t$ (5.17).

ii) Rotation frequency description. $W(t)$ is given by an equa. tion of the type (5.20).

iii) Translational disturbed motion. $V_{P}(t)$ as represented by an equation of the type of (4.8).

iv) Absolute frame composition. $V(t, s)$ as in (5.15).

\section{Recursive Smoothing Receiver}

The previous framework is applied to the passive positioning problem. Given its generality, the receiver is derived and studied in a simpler context, so that its novel features are not clouded by the technical details. The line array and the point source are taken to be fixed. The shape of the array is assumed to be described by the linear class given by (5.12). To be specific, the analysis is presented for the following particular example. The state vector $x(s)$ corresponding to the array description is two dimensional

$$
x(s)=\left[R(s) \mid R^{\prime}(s)\right]^{T}
$$

where $R(s)$ is the separation between the point source and the sensor $s$. The array shape is described by

$$
d x / d s=\left[\begin{array}{ll}
0 & 1 \\
a & 0
\end{array}\right] x(s)+\left[\begin{array}{l}
0 \\
1
\end{array}\right] u(s)
$$

In (5.22), $u(s)$ is a white process in the $s$ variable, $x_{0}$ is unknown. If in (5.22), the matrix element $a$ has a bell shape dependence on $s$, it models a line array which almost replicates a nominal linear shape. For simplicity, the parameter $a$ is here assumed constant. Then, (5.22) models an array which has a nominally hyperbolic sine/cosine geometric shape.

The receiver is decomposed in two blocks as in Fig. 2; see Section II. It is recalled from the discussion following the block decomposition of Fig. 2, that the first block, the Doppler demodulator, depends on the signal temporal structure. Here, solely the study of the geometry reconstructor is considered. It is derived by application of recursive techniques. From Fig. 2, the input to the reconstructor is (after the required normalization)

$$
z(s)=C x(s)+w(s), \quad s \in L
$$

where $C=\left[\begin{array}{ll}0 & 1\end{array}\right]$. The measurement (5.23) corresponds to noisy spatial observations of the Doppler $R^{\prime}(s)$. This is the continuous parameter analog of the relative delays between elements of a discrete array. The positioning problem is reduced to the construction of the "best" estimate (with respect to a given criterion) of $x(s)$ given by (5.22) and (5.23).

At first sight, the linear model (5.22) and (5.23) may seem quite unrealistic from a practical view point. However, two comments will underline its relevance for the passive location problem. First, it is noted that, because the model is linear, the application of the estimation algorithms is straightforward, involving no approximations. Thus one obtains insight on the effects of the sensor uncertainty on the passive location question. The second point is conceptually more delicate. It relates to the fact that, with the model (5.22) and (5.23), the bearing angle is not observable. With a geometrically linear array, this observability comes into the model through the dynamical description of the array shape. For linear shaped arrays, the polar differential description requires a four-dimensional coupled range/bearing state equation. The range and bearing observability is obtained at the cost of using a more complex model that requires higher order nonlinear coupled differential equations. The model (5.22) and (5.23) still preserves the basic question of range determination from Doppler measurements (or, as commented above, from the relative delays between the sensors of a discrete array), addressing it in a very simple theoretical framework. As will be seen from the simulation studies, this simple model quite illustrates the expected difficulties underlying the behavior of the passive positioning receiver. It does so, at the cost of uncoupling it (through the assumption of an hyperbolic shaped array) from the bearing estimation problem. The more general context may be pursued by applying to the nonlinear model the corresponding filtering techniques (e.g. EKB filters, or optimal nonlinear filters, as in [6]).

In summary, the simplified linear model (5.22) and (5.23), preserving the important features of global range determination from passive measurements (range spatial Doppler), serves the basic purpose of illustration of the application of the theory of recursive smoothing to passive location problems. The analysis of the receiver for this particular example also gives important insight on the behavior of the passive locator for more sophisticated and realistic models. 
To construct an estimate of the state $x(s)$ from the measurements (5.23), it is noted that these observations correspond to a fixed interval $L$ (total length of the line array). Also, one is interested in the estimate of $x(s)$ with respect to a reference sensor. Usually, this reference point of the array is its geometric center. The estimation problem in the $s$ variable contrasts then with the corresponding tracking of source motion in the time $t$ parameter, where one is interested in estimating the state using all available measurements up to the present (filtering). The problem of estimating the state vector at a point intermediate to a span of measurements is a smoothing problem. This is a noncausal processing. There are available in the literature alternative implementations of the fixed interval recursive linear smoother [8]-[10]. Here, the formulation of [9], and [10] is adopted. Basically, it consists of two Kalman-Bucy filters (KBF), one running forward (from the left end $L_{i}$ of the array to the right) and the other running backwards (from the right end $L_{0}$ to the left). The forward filter uses the measurement span $\left[L_{i}, s\right]$ to construct the estimate $x_{f}(s)$ of $x(s)$. This is sometimes emphasized by the notation $x_{f}(s \mid s)$. The backward filter uses the data record $\left[s, L_{0}\right]$ to construct the estimate $x_{b}(s)$ of $x(s)$. Often, the notation $x_{b}(s \mid s)$ is used.

The original two-filter smoothing setup of [9] and [10] presented conceptual difficulties. The problem resided on the correct formulation of a backward and reversed Markovian representation of the processes. These difficulties have been clarified [11]-[13]. The continuous parameter framework can then be formulated in symmetrized format. Although it uses twice the measurement at point $s$, this poses no difficulties. With the discrete array, sensor $s$ must only be used once. An asymmetry is built into the problem. These technicalities are not further pursued here, the reader being referred to the references.

Since the Kalman-Bucy algorithm is widely known in the literature, only the backward filter equations are written here. Given the system matrices $A(s), B(s)$, and $C(s)$ (see (5.12), (5.22), and (5.23)), the driving noise covariance matrix $Q(s)$, and the measurement noise covariance matrix $R(s)$, the backward filter is

$$
-d x_{b}(s) / d s=-A(s) x_{b}(s)+K_{b}(s)\left[y(s)-C(s) x_{b}(s)\right] .
$$

In (5.24), the matrix gain $K_{b}(s)$ is

$$
K_{b}(s)=P_{b}(s) C^{T}(s) R^{-1}(s)
$$

where $P_{b}(s)$ is the error covariance associated with $x_{b}(s)$, given by the solution of the nonlinear matrix differential Riccati equation

$$
\begin{aligned}
-d P_{b}(s) / d s= & -A(s) P_{b}(s)-P_{b}(s) A^{T}(s)+Q(s) \\
& -P_{b}(s) C^{T}(s) R^{-1}(s) C(s) P_{b}(s) .
\end{aligned}
$$

These equations are initialized at

$$
P_{b}^{-1}\left(L_{0}\right)=0
$$

and

$$
\lim _{s \rightarrow L_{0}} P_{b}^{-1}(s) x_{b}(s)=0
$$

In fact, interpretation of condition (5.27) shows that $x_{b}(s)$ as given by (5.24)-(5.28) is an estimate (no a priori knowledge is assumed when constructing this estimate); see [13] for comments on this issue. Condition (5.28) requires that the filter be implemented as an "information filter," see for example [14].

The smoothed Bayesian estimate $x(s \mid s)$ and its associated error covariance matrix $P(s / L)$ are obtained by suitably combining the forward estimate $x_{f}(s)$ and its covariance error matrix $P_{f}(s)$ and the backward estimate $x_{b}(s)$ and its error covariance matrix $P_{b}(s)$. The combining formula is well known from statistics

$$
\begin{aligned}
& x(s \mid L) P(s \mid L)\left[P_{f}^{-1} x_{f}+P_{b}^{-1} x_{b}\right], \quad s \in L \\
& P(s \mid L)=\left[P_{f}^{-1}+P_{b}^{-1}\right]^{-1}, \quad s \in L .
\end{aligned}
$$

In (5.29) and (5.30), explicit dependence on $s$ has been partially omitted.

The two-filter implementation of the optimal linear recursive smoother has been simulated in a digital computer. The processes $u(s)$ and $w(s)$ have variance parameters $q$ and $r$, respectively. The total length of the line array is $L$. The following figures show, for different conditions, the behavior of the elements of $P(s)=P_{f}(s), P_{b}(s)$, and $P(s \mid L)$. In Fig. 13, $L$ is kept fixed, and the statistical parameters $q$ and $r$ are changed. The set of curves study the behavior of the first element, i.e., the range error covariance, of each of the matrices $P_{f}, P(s \mid L)$, and $P_{b}$ as a function of the reference point $s$ of the array. It is interesting to note the monotonic behavior of $P_{11}(s)=P_{f 11}(s)$, and $P_{b 11}(s)$. Its combined value, according to (5.30), has, however, a convex cup shape, with a minimum at the interior of the array. This indicates that positioning with respect to an interior point has smaller error variance than with respect to the extremes of the array. The array length $L$ has been chosen large enough, so that a steady-state behavior is exhibited in both the forward and backward filters' Riccati equations. To emphasize this steady-state error behavior, Fig. 14 shows $P_{11}(s \mid L)$ as a function of $s$ for different values of $L$. The $\min P_{11}(s \mid L)$ monotonically decreases to a threshold; see Fig. 15. This contrasts with the usual integral formulations of the passive location problems. In these, the error performance improves monotonically with the array length. Here, because of the error uncertainty associated with the location of the array sensors, there is a minimum in the error performance (steady-state behavior of the Riccati equation). There is no point in increasing the array length beyond a given total length.

The set of curves of Fig. 13, also show the variation of $P_{11}(s \mid L)$ with the statistical parameters $q$ and $r$. To study the sensitivity of the passive recursive smoother to the a priori knowledge, i.e., to the uncertainty $P_{f}(0)$ associated with the initial condition of the smoother, Fig. 16 studies, for four sets of widely different initial variances $P_{11}(0)$, the mean-square- 


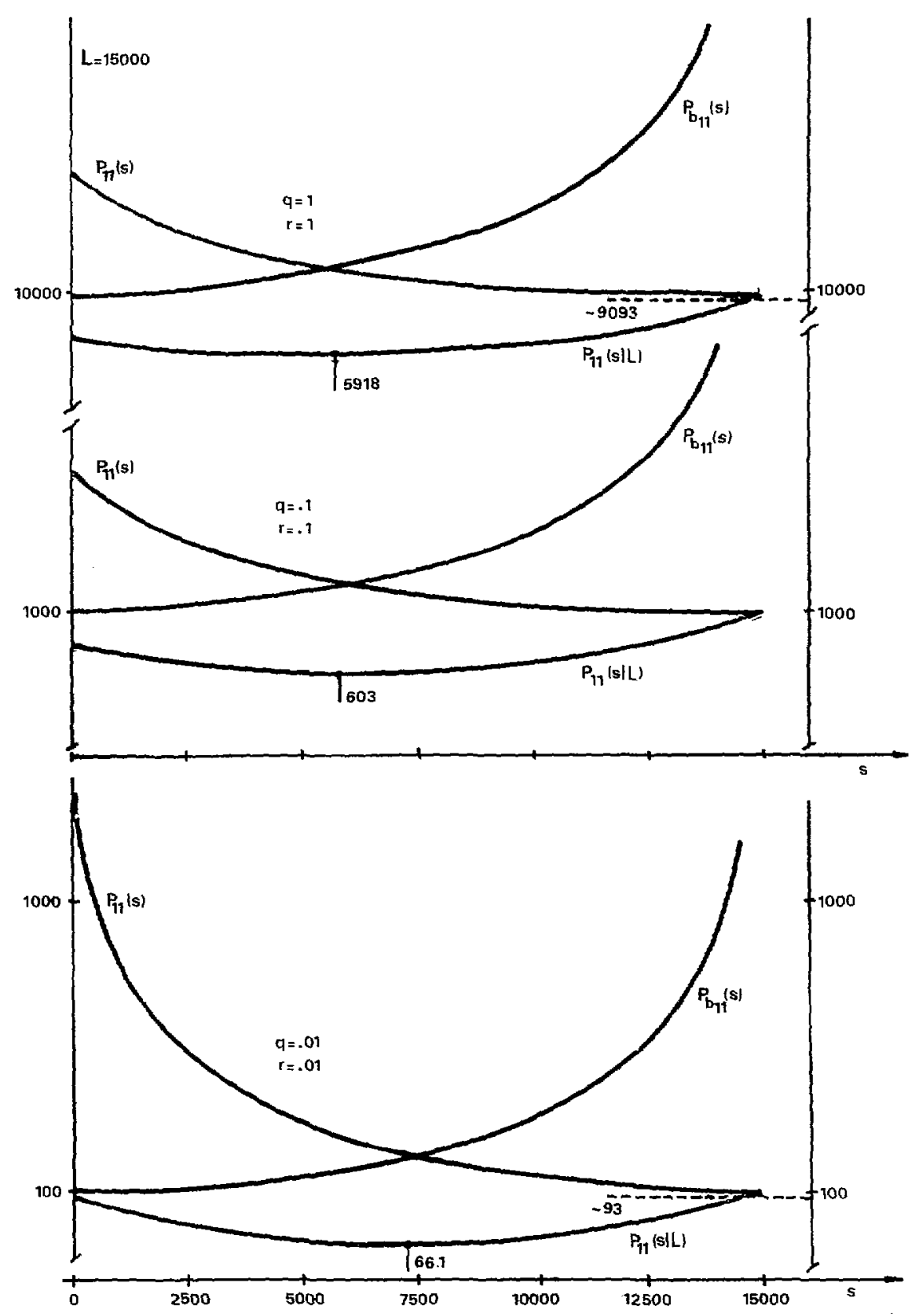

Fig. 13. Study of the mean-square range error for the passive locator: Forward $\left(P_{11}(s)\right)$, backward $\left(P_{b 11}(s)\right)$, and smoothing $\left(P_{11}(s \mid 1)\right)$ variances $\left(\mathrm{ft}^{2}\right)$ as a function of $s(\mathrm{ft}) . L=$ total array length (ft), $q=$ sensor uncertainty noise level, $r=$ Doppler measurement noise level. 


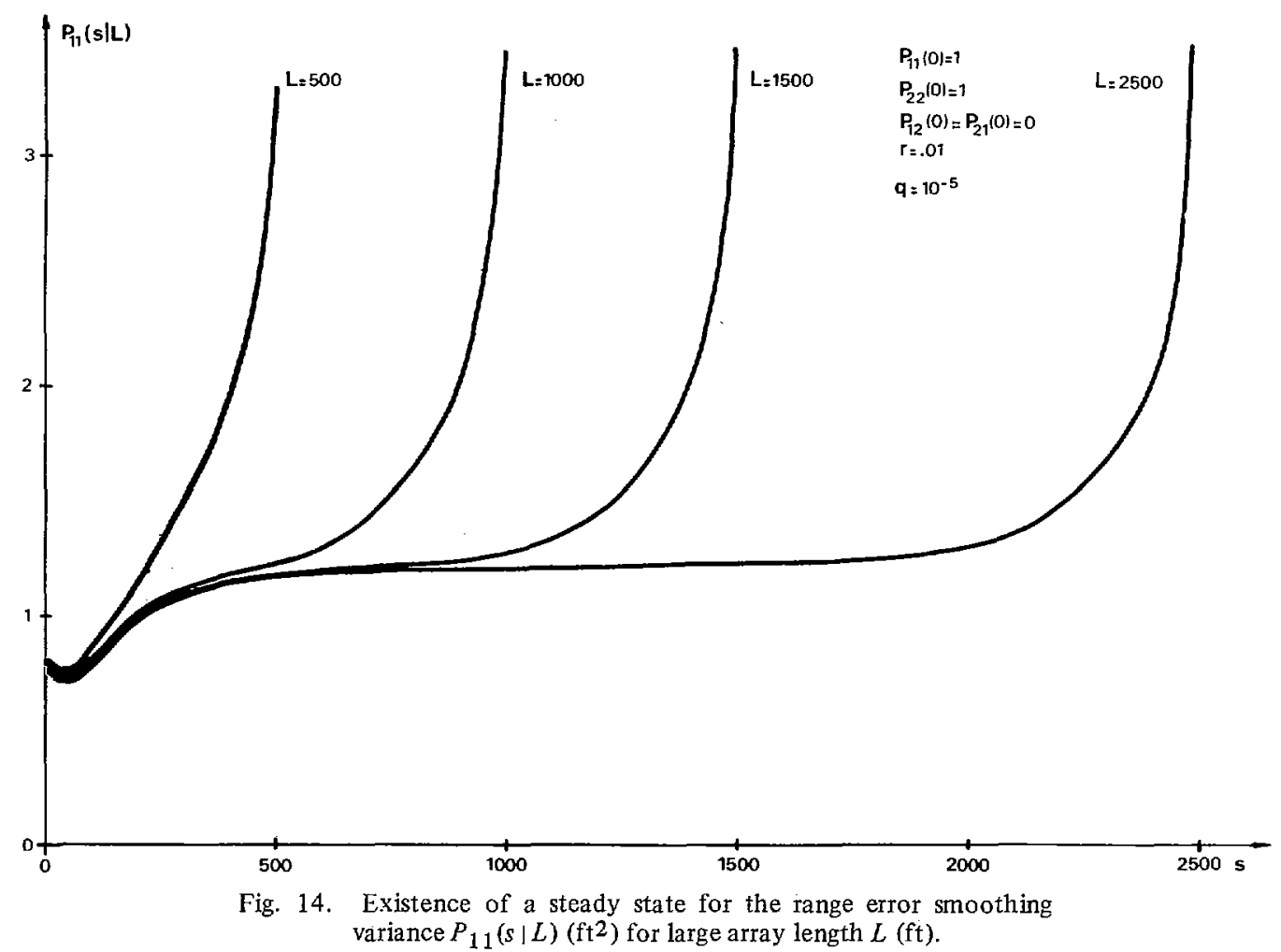

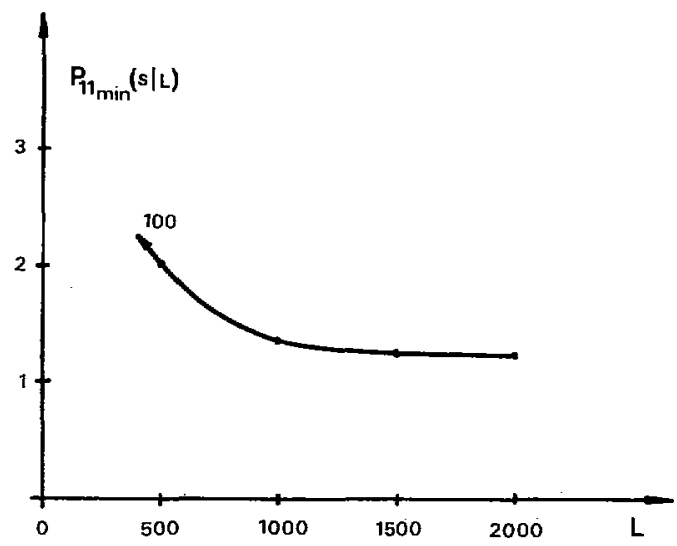

Fig. 15. Study of the minimum of the range error smoothed variance $P_{11 \mathrm{~min}}(\mathrm{~s} \mid L)\left(\mathrm{ft}^{2}\right)$ as a function of the array length $L(\mathrm{ft})$.

error behavior of the linear recursive smoother. The conclusion is that, excluding the unrealistic situation where the initial uncertainty is smaller than the steady-state min $P_{11}(s)$, the passive locator is quite insensitive to the initial conditions. This robustness to the a priori knowledge of the recursive smoother is quite important in practical applications. It is also emphasized that by combining the forward and backward estimates according to (5.27) and (5.28), a significant improvement is obtained with respect to each of the individual estimates. In fact, the best the forward filter can do is at the extreme right of the array, after having processed the whole data span $L$. This, of course, assumes that the array is sufficiently large, so that steady state has been reached. At this point, due to (5.27), the forward filter and the linear smoother exhibit identical performances. From all figures, it is apparent that the minimum error performance of the optimal smoother is significantly smaller than its right-end error performance.

\section{CONCLUSION}

The paper has focused on the geometric aspects of the passive positioning problem. The type of formulation chosen to describe these geometry (spatial and temporal) constraints affects significantly the structure of the receiver. Section III has addressed the problem where the geometry and the motions are completely described by a finite number of unknown parameters. This has been termed the integral space/integral time description. The receiver performs an ML estimation of the unknown parameters. The computational complexity and the sensitivity of the receiver to model perturbations have been discussed. In applications where significant dynamics are available, tracking with this ML passive receiver is computationally ineffective. In Section IV, the motions have been modeled using a set of stochastic differential equations in time. The resulting tracking receiver is quite insensitive to the path instabilities of the source motions. Finally, Section $V$ has considered the problem of irregular array shapes and applications where, for example, due to stretching and bending, the exact array configuration is uncertain. A differential description using the arc length $s$ of the array has been introduced. Again, this approach completely dualizes the time and space descriptions, but now in differential terms. It has been noted, however, that the passive receiver is conceptually accomplishing different tasks in time and space. The recursive time tracker is a filter, while the space locator is a recursive smoother. The paper has 


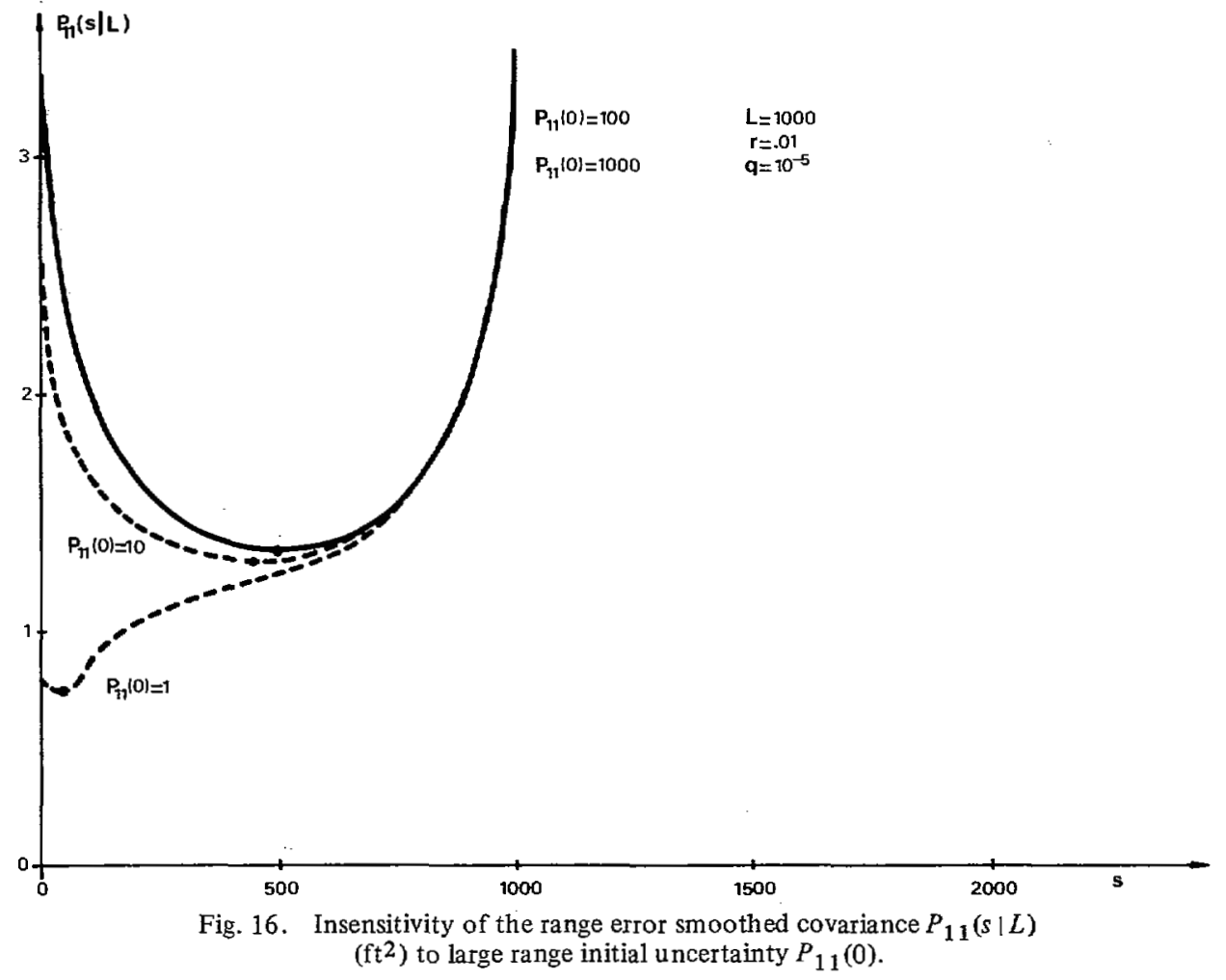

presented quite general differential descriptions for the array. The case of a rigid line array subject to stochastic motions has also been completely described. The optimal linear smoother, implemented in the two recursive filters formulation of [9] and [10], has been simulated. The results from a case study where the model belongs to a linear class have been discussed, providing significant insight to the passive location problem. The same design techniques can be applied to classes of nonlinear models, as long as one performs dynamical linearizations, or then applies nonlinear filtering techniques like in [6] .

\section{REFERENCES}

[1] J. M. F. Moura and A. B. Baggeroer, "Passive systems theory with narrow band and linear constraints: Part I-Spatial diversity," IEEE J. Oceanic Eng., vol. OE-3, no. 1, pp. 5-13, 1978.

[2] J. M. F. Moura, "Passive systems theory with narrow band and linear constraints: Part II-Temporal diversity." IEEE J. Oceanic Eng., vol. OE-4, no. 1, pp. 19-30, 1979.

[3] ___. "Passive systems theory with narrow band and linear constraints: Part III-Spatial/temporal diversity," IEEE J. Oceanic Eng., vol. OE-4, no. 3, pp. 113-119, 1979.

[4] V. H. MacDonald and P. M. Schultheiss, "Optimum bearing estimation in a spatially incoherent noise environment, " $J$. Acoust. Soc. of America, vol. 46, no. 1, pt. 1, pp. 37-43, 1969.

[5] R. S. Bucy, C. Hecht, and K. D. Senne, "An engineer's guide to building nonlinear filters, "' Frank J. Seiler Res. Lab., Tech. Rep. SRL-TR-72-0004, May 1972.

[6] J. M. N. Leitão and J. M. F. Moura, "Nonlinear filtering in phase acquisitions," in Signal Processing Theories and Applications, M. Kunt and F. de Coulon, Eds. Amsterdam, The Netherlands: North Holland, 1980, pp. 437-442.

[7] J. M. F. Moura, "The hybrid algorithm: A solution to acquisition and tracking," J. Acoust. Soc. of America, vol. 69, no. 6, pp. 1663-1672, June 1981

[8] H. E. Rauch, F. Tung, and C. T. Striebel, "On the maximum likelihood estimates for linear dynamic systems," AIAA J., vol. 3 , no. 8, pp. 1445-1450, Aug. 1965.

[9] D. Q. Mayne, "A solution to the smoothing problem of linear dynamic systems," Automatica, vol. 4, no. 2, pp. 73-92, Nov. 1966.

[10] D. C. Fraser and J. E. Potter, "The optimum linear smoother as a combination of two optimum linear filters," IEEE Trans. Automat. Contr., vol. AC-14, no. 4, pp. 387-390, Aug. 1969.

[11] L. Ljung and T. Kailath, "Backward Markovian models for second-order stochastic processes," IEEE Trans. Inform. Theory, vol. IT-22, no. 4, pp. 488-492, July 1976.

[12] G. Verghese and T. Kailath, "A further note on backwards Markovian models," IEEE Trans. Inform. Theory, vol. IT-25, no. 1, pp. 121-124, Jan. 1979.

[13] J. E. Wall, Jr., A. S. Willsky, and N. R. Sandel, Jr., "On the fixed interval smoothing problem,' LIDS Rep., MIT, Cambridge, MA, 1980.

[14] A. Gelb, Applied Optimal Filtering. Cambridge, MA: MIT Press, 1973.

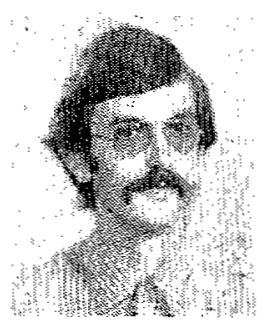

José M. F. Moura (S'70-M'75) was born in Mozambique, in 1946. He received the E.E. degree from Instituto Superior Técnico (IST), Lisboa, Portugal, in 1969, the M.Sc. and E.E. degrees in 1973, and the Sc.D. degree in electrical engineering and computer science in 1975 , all from the Massachusetts Institute of Technology, Cambridge.

In 1975, he rejoined the faculty of the Department of Electrical Engineering of IST where he has been Professor Catedrático since 1979. He has been on the scientific council of IST since 1978, and in the Executive Committee of the Electrical Engineering Department since 1980. He has been the coordinator of the Master of Science program in Electrical Engineering and Computer Science of IST since its establishment in 1981. He teaches courses in control and statistical communication. His research interests lie on the applications of estimation, optimal linear and nonlinear filtering, and systems theories, namely, in guidance and control and, in underwater acoustics. During the Summers of 1978, 1979, 1980, and 1981, he was a Visiting Scholar with the Control Group of the Aerospace Department of the University of Southern California. He has published several papers in international technical journals and presented communications to specialized international meetings. He is coeditor (with R. S. Bucy) of Nonlinear Stochastic Problems (Amsterdam, The Netherlands: D. Reidel, 1983).

Dr. Moura is a member of SIAM, Sigma Xi, the Institute of Navigation, the New York Academy of Sciences, and Ordem dos Engenheiros. He has been cited in the 6th edition of Marquis' Who' Who in the World. 